University of Wollongong

Research Online

Faculty of Engineering and Information

Faculty of Engineering and Information

Sciences - Papers: Part A

Sciences

$1-1-2015$

\title{
Alleviation of neutral-to-ground potential rise under unbalanced allocation of rooftop pv using distributed energy storage
}

Md Jan E Alam

University of Queensland, mjealam@uowmail.edu.au

Kashem M. Muttaqi

University of Wollongong, kashem@uow.edu.au

Danny Sutanto

University of Wollongong, soetanto@uow.edu.au

Follow this and additional works at: https://ro.uow.edu.au/eispapers

Part of the Engineering Commons, and the Science and Technology Studies Commons

Research Online is the open access institutional repository for the University of Wollongong. For further information contact the UOW Library: research-pubs@uow.edu.au 


\title{
Alleviation of neutral-to-ground potential rise under unbalanced allocation of rooftop pv using distributed energy storage
}

\author{
Abstract \\ A high penetration of one-phase rooftop solar photovoltaic (PV) units with unbalanced allocation can \\ create considerable neutral current and neutral potential rise in low voltage (LV) four-wire multigrounded \\ distribution networks, even with balanced loads. Because of the limitations of traditional strategies to \\ mitigate the combined effect of load and PV unbalance, this paper proposes the use of distributed energy \\ storage to reduce the neutral current and neutral potential under a high penetration of unbalanced rooftop \\ solar PV allocation. A power-balancing algorithm based on charge/discharge control is developed to \\ continuously adjust the power exchange with the grid to mitigate the neutral current and neutral potential \\ rise, while minimizing power drawn from the energy storage. A dynamic model of the integrated PV- \\ storage system is developed to investigate the dynamic performance of the proposed strategy. An \\ Australian LV distribution system is used to verify its performance and the results are presented

\section{Keywords} \\ rooftop, allocation, distributed, energy, pv, storage, unbalanced, under, alleviation, neutral, ground, \\ potential, rise

\section{Disciplines} \\ Engineering | Science and Technology Studies

\section{Publication Details} \\ M. J E. Alam, K. M. Muttaqi \& D. Sutanto, "Alleviation of neutral-to-ground potential rise under unbalanced \\ allocation of rooftop pv using distributed energy storage," IEEE Transactions on Sustainable Energy, vol. 6, \\ (3) pp. 889-898, 2015.
}




\title{
Alleviation of Neutral-to-Ground Potential Rise under Unbalanced Allocation of Rooftop PV Using Distributed Energy Storage
}

\author{
M. J. E. Alam, Member, IEEE, K. M. Muttaqi, Senior Member, IEEE and D. Sutanto, Senior Member, \\ IEEE
}

\begin{abstract}
A high penetration of 1-phase rooftop solar photovoltaic (PV) units with unbalanced allocation can create considerable neutral current and neutral potential rise in low voltage (LV) 4-wire multi-grounded distribution networks, even with balanced loads. Because of the limitations of traditional strategies to mitigate the combined effect of load and PV unbalance, this paper proposes the use of distributed energy storage to reduce the neutral current and neutral potential under a high penetration of unbalanced rooftop solar PV allocation. A power balancing algorithm based on charge/discharge control is developed to continuously adjust the power exchange with the grid to mitigate the neutral current and neutral potential rise, while minimizing power drawn from the energy storage. A dynamic model of the integrated PV-storage system is developed to investigate the dynamic performance of the proposed strategy. An Australian LV distribution system is used to verify its performance and results are presented.
\end{abstract}

Index Terms-- Rooftop solar PV, 4-wire multi-grounded LV network, neutral current, neutral potential rise, distributed energy storage, charging/discharging.

\section{INTRODUCTION}

$\mathrm{N}$ EUTRAL current flow in low voltage (LV) 4-wire multigrounded distribution networks is a known problem among distribution system research community and protection engineers. The fundamental frequency component of the neutral current is mainly produced by load unbalance [1] and unbalanced impedances of the network. The unbalanced phase currents do not sum up to zero, and the resultant neutral current flows through the neutral conductor and neutral grounding resistances causing health and safety concerns. Harmonic components produced by the magnetization of distribution transformers and non-linear loads can produce triplen harmonics, which can increase the neutral current to be even higher than the phase current [1]. In a healthy distribution system, the neutral grounding resistances would be small and therefore, a reasonable amount of neutral current would not increase the neutral potential significantly. However, if the neutral grounding resistances increase, for

This work is supported by the Australian Research Council (ARC) and Essential Energy Linkage Grant, LP100100618.

M. J. E. Alam, K. M. Muttaqi and D. Sutanto is with Australian Power Quality and Reliability Center, School of Electrical Computer and Telecommunications Engineering, University of Wollongong, NSW 2522, Australia (e-mail: mjea982@uowmail.edu.au, kashem@uow.edu.au, soetanto@uow.edu.au). example due to bonding of the earth wire with a non-metallic water piping system [2], the neutral potential or Neutral-toGround Voltage (NGV) can become significant. A high NGV value can damage sensitive electronic and computing equipment due to the common-mode noise effect [3]. Manufacturers of computing equipment have specified $0.5 \mathrm{~V}$ [3] as the limit of common-mode noise; a more stringent limit of $0.1 \mathrm{~V}$ is reported in [4]. NGV and other forms of stray voltages are also detrimental for human beings [5] and farm animals [6]. To ensure a safe and reliable operation of distribution networks, neutral current and NGV rise have to be treated with due importance.

A high penetration of single phase rooftop solar PV units with an unbalanced allocation has the potential to worsen the classical neutral current and neutral potential problem. Distribution utilities are not always in a position to directly control the generation of solar PV units at different phases of a network. Therefore, a highly unbalanced allocation of solar PV units can create a significantly large neutral current that can cause the NGV to exceed the acceptable limit.

Several methods for the mitigation of neutral current and neutral potential rise have been reported in the literature [610]. The balancing of loads $[7,8,10]$ is a traditional mitigation method used for the reduction of neutral current produced by the load unbalance, although in practice it is very difficult to have $100 \%$ load balance. Other forms of traditional mitigation techniques, such as the re-sizing of neutral conductor $[7,10]$, improving grounding system [7, 10], adding passive harmonic filter, or installation of equipotential planes [6, 7, 9], may need significant additional material and can increase installation costs. Further, the variability of solar PV generation would make it difficult to balance the combined effect of load and solar PV unbalance using the traditional mitigation techniques that are mainly of "static" type, and does not change with the variation of solar PV output. Therefore, new mitigation strategies need to be developed to balance out the combined unbalance produced by rooftop solar PV units and load demands.

Mitigation of voltage unbalance using optimal control of PV inverter active and reactive power was proposed in [11]. Active filters [12] have also been proposed to reduce the harmonics and hence can reduce the triplen harmonics flowing in the neutral current; however such a filter cannot compensate for active power variation such as PV output fluctuation. 
Distributed energy storage has been proposed to solve renewable energy related issues in LV networks, such as voltage rise [13], power quality issues, and also for peak shaving. When available, such energy storage system can be also applied for voltage unbalance mitigation by charging and discharging of PV power as shown in [14], and hence can increase the benefit of the storage device. In a previous work [15], the authors have explored the application of distributed energy storage devices integrated with rooftop PV systems for the mitigation of neutral current and NGV problems. However, the detailed method for charging/discharging control, including its dynamic response, was not investigated in [15].

As an extension of the work reported in [15], the main contributions of this paper are: (i) to develop a power balancing algorithm for mitigating the neutral current and NGV rise problems while minimize the power consumption/delivery by the energy storage; and, (ii) to develop a charging/discharging strategy using the proposed power balancing algorithm to provide a controlled mitigation effect under variable load and PV unbalance. Given the safety and power quality issues of neutral voltage rise caused by current unbalance, this paper will contribute towards managing this problem. In addition, a dynamic model of the $\mathrm{PV}$-storage integrated system is developed to investigate the performance of the proposed strategy under the time lags associated with power electronic systems and battery storage. The proposed approach will be analyzed for their effectiveness in mitigating the neutral current and neutral potential problems in the context of Australian LV distribution systems.

\section{Neutral CurRent AND Neutral Potential CREATED BY UNBALANCED ALLOCATION OF ROOFTOP SOLAR PV UNITS}

The neutral current produced at any given bus of a three phase 4-wire multi-grounded LV feeder, as shown in Fig. 1, can be obtained from the expression in (1).

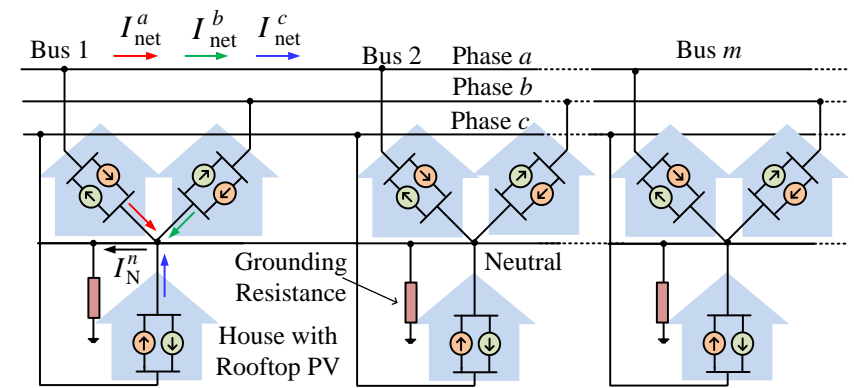

Fig. 1. Neutral current in a 4-wire multigrounded LV feeder produced by unbalanced load and PV allocation.

$$
\begin{aligned}
& I_{\mathrm{N}}^{n}=-\left(I_{\mathrm{net}}^{a}+I_{\mathrm{net}}^{b}+I_{\mathrm{net}}^{c}\right) \\
& I_{\mathrm{net}}^{p}=\frac{\left(P_{\mathrm{L}}^{p}-P_{\mathrm{PV}}^{p}\right)-j\left(Q_{\mathrm{L}}^{p}-Q_{\mathrm{PV}}^{p}\right)}{\left(V_{\mathrm{re}}^{p}-V_{\mathrm{re}}^{n}\right)-j\left(V_{\mathrm{im}}^{p}-V_{\mathrm{im}}^{n}\right)}, p \in\{a, b, c\}
\end{aligned}
$$

where, $I_{\mathrm{N}}^{n}$ is the total neutral current at the given node; $I_{\text {net }}^{a}, I_{\text {net }}^{b}$, and $I_{\text {net }}^{c}$ are the net currents in phase $a, b$ and $c ; P_{\mathrm{L}}$, $P_{\mathrm{PV}}$ are the active load and active power of the PV inverter at the respective phases; $Q_{\mathrm{L}}, Q_{\mathrm{PV}}$ are the reactive power of the load, and reactive power of the PV inverter at the respective phases; $V_{\text {re }}$ and $V_{\text {im }}$ are the real and imaginary components of the respective phase and neutral voltages.

From (1) it is evident that the neutral current can still be produced even if the loads are fully balanced. To investigate how solar PV unbalance can create neutral current and voltage problems, the feeder in Fig. 1 is considered with the loads perfectly balanced. The PV outputs in phase $a$ are varied from zero to twice the outputs in phase $b$ and $c$ using a scaling factor $\lambda$ and its effect on the neutral current and voltage is observed in Fig. 2. The Current Unbalance Factor (CUF) defined in [16] is used to measure the current unbalance created by PV unbalance.

$$
C U F=\frac{\sqrt{\left|I_{\mathrm{ns}}\right|^{2}+\left|I_{\mathrm{zs}}\right|^{2}}}{\left|I_{\mathrm{ps}}\right|} \times 100 \%,\left[\begin{array}{c}
I_{\mathrm{ps}} \\
I_{\mathrm{ns}} \\
I_{\mathrm{zs}}
\end{array}\right]=\frac{1}{3}\left[\begin{array}{ccc}
1 & a & a^{2} \\
1 & a^{2} & a \\
1 & 1 & 1
\end{array}\right], a=e^{j\left(\frac{2 \pi}{3}\right)}(2
$$

where, $I_{\mathrm{ps}}, I_{\mathrm{ns}}$ and $I_{\mathrm{zs}}$ are positive, negative and zero sequence currents, respectively.

The left and right hand side compass plots in Fig. 2(a) correspond to the cases with $\lambda=0$ and $\lambda=2$, respectively, that represents two extreme cases of current unbalance (CUF: $400 \%$ and $70 \%$ ) and therefore shows high neutral current. The middle compass plot corresponds to the case with $\lambda=1$, where the net currents are balanced and the neutral current is approximately zero.
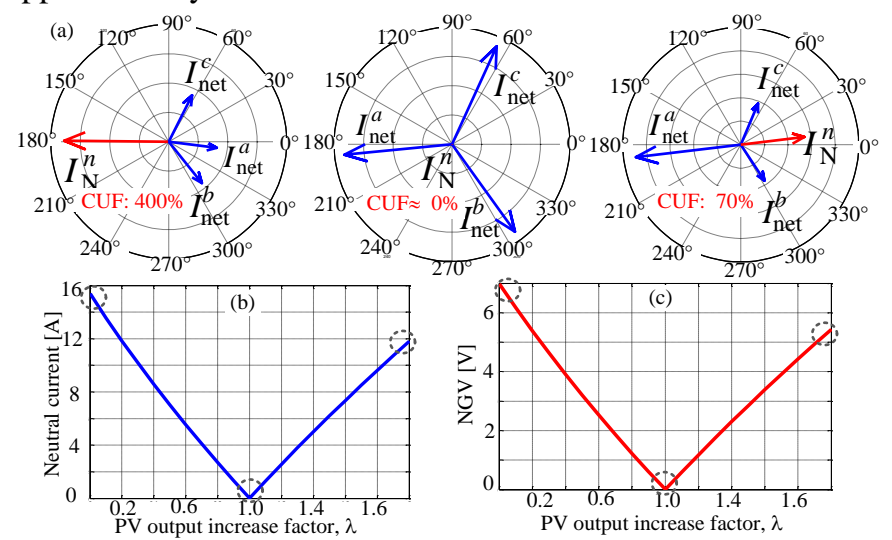

Fig. 2. Effect of PV unbalance on neutral current and voltage. (a) The vector diagrams of net currents and neutral current. (b) Neutral current. (c) NGV.

The profiles of the neutral current and NGV as $\lambda$ increases are shown in Fig. 2(b) and (c), respectively. The neutral current and NGV value increases for $0<\lambda<1$ and $1>\lambda>2$, with a theoretically zero value when PV outputs are balanced (i.e. $\lambda=1$ ). If the loads are also unbalanced, this may further aggravate the neutral current and neutral potential rise created by the unbalanced allocation of the PV units.

The neutral current produced by an unbalanced allocation of rooftop solar PV would be significant during midday due to a high PV output. A real evidence of the midday increase of the neutral current caused by unbalanced PV generation in a distribution feeder was captured by an Australian utility, shown in Fig. 3. In a previous work [17], authors developed a power flow approach for integrated 3-wire and 4-wire multigrounded networks and investigated the PV impact on an Australian distribution system where the neutral grounding resistances in the LV feeders are elevated because of the 
bonding of the earth wire with the non-metallic water reticulation system [2]. The results show that the neutral voltage can exceed the acceptable level of $0.5 \mathrm{~V}$ [3] in a feeder with an unbalanced allocation of PV, as shown in Fig. 4. The current unbalance factors determined using (2) are also found high during the periods of high neutral voltage, as shown in Fig. 4.

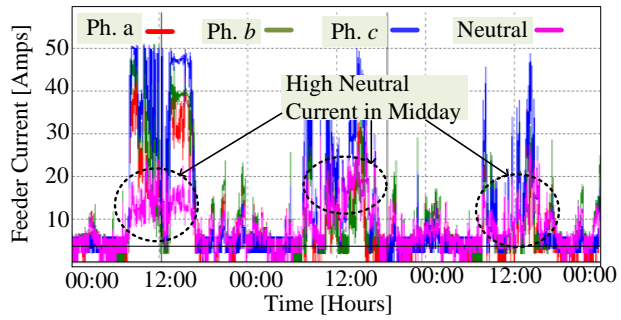

Fig. 3. Captured unbalanced phase currents and neutral current profiles in a distribution feeder in Australia.

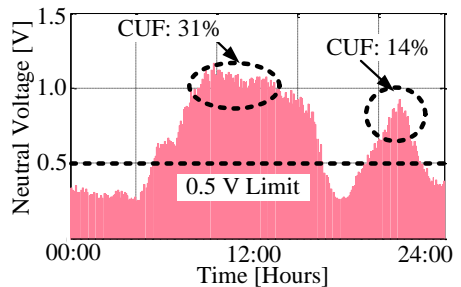

Fig. 4. Simulated neutral voltage in an Australian distribution system caused by unbalanced rooftop PV allocation.

To reduce a potentially high $\mathrm{NGV}$ produced by the combined effect of variable 1-phase PV outputs and unbalance load demands, a dynamic mitigation strategy using energy storage is developed in the following section.

\section{Proposed Distributed Energy Storage Based MITIGATION STRATEGY}

In this strategy, battery storage devices already integrated with the PV systems in the feeder are used; a schematic of the proposed arrangement is shown in Fig. 5. Each of the households of a given phase in a given bus is connected to the households in the other two phases in the same bus through a communication link. With the progress of smart grid technologies extending up to the low voltage customer level, such arrangements would be possible. In this paper, it is assumed that the communication link is reliable and therefore, always available. It is important to consider the latency (the time taken for a packet of data to be transferred between two points in the network) and the bandwidth (the data transfer rate) implications while deploying the available communication arrangement for the proposed strategy. The Quality of Service (QoS) requirements [18, 19] of standard smart grid technologies need the latency to be within the range of a few milliseconds to a few tens of milliseconds depending on the application. The bandwidth will depend on the latency, the message length and number of messages per second. An example of bandwidth calculation performed in [20] for the communication system in a 10,000 feeder smart grid connecting 1 million customers evaluated the required bandwidth to be $100 \mathrm{Mbps}$ considering $10 \mathrm{~ms}$ latency, $100 \mathrm{bits}$ message and 1 million messages per second. As the proposed strategy only intends to communicate among a few households at the three phases of a given bus, any standard smart grid communication bandwidth would be sufficient.

As the proposed strategy will use the regular power connection between the storage device and the PV system, the standard fault/short circuit protection facilities available in an integrated PV (grid-tied) and energy storage system would be suitable.

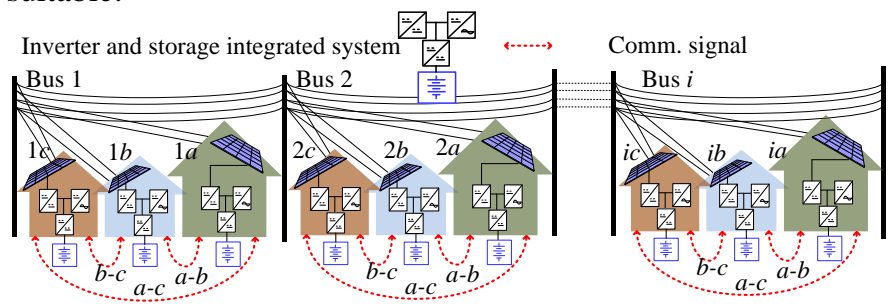

Fig. 5. Schematic diagram of the proposed distributed storage based strategy for mitigation of $\mathrm{NC}$ and $\mathrm{NGV}$ problems.

For balancing operation, it is intended to charge/discharge the storage devices such that the power exchange with grid (reverse power flow during high PV period or forward power flow during low or no PV period) becomes equal for all the three phases at each bus in the LV feeder. A method to determine the amount of power to be consumed or delivered by each of the storage device for the balancing operation is developed below.

\section{A. Development of a Power Balancing Algorithm based on Charging/Discharging of Distributed Storage}

Assume that the power to be exchanged with the grid ( $\left.P_{\text {grid }}\right)$ at each of the phases at the $i$-th bus will have to be made equal to a reference power $P_{\text {grid }}^{\text {ref }-i}$. The amount of power to be consumed or delivered by the storage device at each of the phases for this purpose can be obtained as given below.

$$
\left[\begin{array}{l}
P_{\mathrm{DES}}^{i a} \\
P_{\mathrm{DES}}^{i b} \\
P_{\mathrm{DES}}^{i c}
\end{array}\right]=P_{\text {grid }}^{\text {ref }-i}-\left[\begin{array}{c}
P_{\mathrm{grid}}^{i a} \\
P_{\text {grid }}^{i b} \\
P_{\text {grid }}^{i c}
\end{array}\right]=P_{\text {grid }}^{\text {ref }-i}-\left[\begin{array}{c}
P_{\mathrm{L}}^{i a}-P_{\mathrm{PV}}^{i a} \\
P_{\mathrm{L}}^{i b}-P_{\mathrm{PV}}^{i b} \\
P_{\mathrm{L}}^{i c}-P_{\mathrm{PV}}^{i c}
\end{array}\right]
$$

where, $P_{\mathrm{DES}}$ is the power to be consumed or delivered by the distributed energy storage for balancing operation, $P_{\mathrm{L}}$ is the load active power, $P_{\mathrm{PV}}$ is the inverter active power, all for the respective phases at the $i$-th bus, as shown in Fig. 5 .

If all the phases experience reverse power flow (negative $P_{\text {grid }}$, during high PV period), then the balancing is performed by the charging operation, as shown in Fig. 6(a), whereas, balancing is performed by the discharging operation if power is drawn from the grid at all the phases (positive $P_{\text {grid, }}$, during low or no PV period), as shown in Fig. 6(b).

The power used for the balancing operation needs to be minimized to reduce the amount of storage capacity required. The value of $P_{\text {grid }}^{\text {ref }-i}$ that minimizes the total amount of power usage from the three distributed storage devices in a bus can be obtained by solving the constrained minimization problem formulated below.

$$
\text { minimize : } \sum_{p \in\{a, b, c\}}\left|P_{\mathrm{DES}}^{i p}\right| \text {, subject to.: }
$$




$$
\begin{gathered}
{\left[\begin{array}{l}
0 \\
0 \\
0
\end{array}\right]=-\left[\begin{array}{ccc}
1 & -1 & 0 \\
0 & 1 & -1 \\
-1 & 0 & 1
\end{array}\right]\left[\begin{array}{l}
P_{\mathrm{DDS}}^{i a} \\
P_{\mathrm{DDS}}^{i b} \\
P_{\mathrm{DES}}^{i c}
\end{array}\right]+\left[\begin{array}{l}
P_{\text {grid }}^{i a}-P_{\text {grid }}^{i b} \\
P_{\text {grid }}^{i b}-P_{\text {grid }}^{i c} \\
P_{\text {grid }}^{i c}-P_{\text {grid }}^{i a}
\end{array}\right]} \\
\left(P_{\mathrm{DES}}^{i a} \times P_{\mathrm{DES}}^{i b}\right) \geq 0,\left(P_{\mathrm{DES}}^{i b} \times P_{\mathrm{DES}}^{i c}\right) \geq 0,\left(P_{\mathrm{DES}}^{i c} \times P_{\mathrm{DES}}^{i a}\right) \geq 0
\end{gathered}
$$
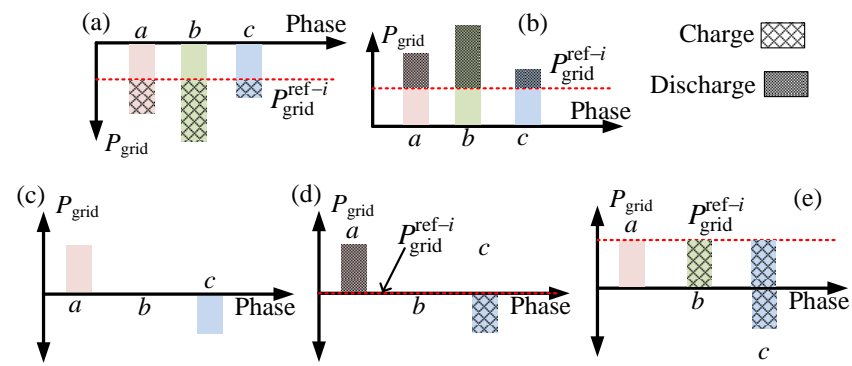

Fig. 6. Grid power exchange scenarios (a) Pgrid at all phases $<0$. (b) Pgrid at all phases $>0$. (c) Mixed type of Pgrid. (d) Balancing by charging and discharging. (e) Balancing by only charging.

The equality constraint in (4b) ensures that an equal power exchange with the grid at all the phases (power balancing) and the inequality constraints in $(4 \mathrm{c})$ ensures the correct decision is made on the storage operation (charging or discharging). According to the solutions obtained from (4), if all the phases experience the same type of power exchange with the grid (either reverse power or forward power), the total power for the balancing operation at a given $i$-th bus can be minimized when $P_{\text {grid }}^{\text {ref }-i}$ is obtained using (5).

$$
P_{\text {grid }}^{\text {ref }-i}=\min \left(\left|P_{\text {grid }}^{i a}\right|,\left|P_{\text {grid }}^{i b}\right|,\left|P_{\text {grid }}^{i c}\right|\right)
$$

However, depending on the load demand and the solar PV generation, a bus may experience a mixed type of $P_{\text {grid. }}$ For example in Fig. 6(c), phase $a, b$ and $c$ have positive (+ve), 0 and negative (-ve) $P_{\text {grid, }}$ respectively. Balancing can be performed using a combination of charging and discharging operation as shown in Fig. 6(d), or using either of those; balancing by charging only is shown in Fig. 6(e). For these cases, (4) needs to be solved to select the option that ensures the sum of the power consumption or delivery by the storage devices in a bus is the minimum. If the PV system in a phase is faulty or a phase does not have a PV system, then instead of having a reverse power flow, it will rather have a forward power flow. If it is intended to provide a balancing effect in these cases, (4) needs to be solved to determine the appropriate amount of charging/discharging power. Whether there will be any storage without a PV is a different concern and is not considered in this paper. The proposed strategy however would be most optimal when each of the customers has a PV system integrated with a storage device in every phase.

Once $P_{\mathrm{DES}}$ is obtained using (3)-(5), the charging/discharging rate of the battery at the phase ' $p$ ' of the $i$-th bus can be obtained using the expression below.

$$
I_{\mathrm{DES}}^{i p}=\frac{P_{\mathrm{DES}}^{i p}}{V_{\mathrm{DES}}^{i p}}=\frac{P_{\mathrm{DES}}^{i p}}{f\left(\mathrm{SoC}_{\mathrm{DES}}^{i p}, C_{\mathrm{DES}}^{i p}, \mathbf{p}_{\mathrm{DES}}^{i p}\right)}
$$

where, $I_{\mathrm{DES}}^{i p}$ is the charging/discharging rate, $p \in\{a, b, c\}$,
$V_{\mathrm{DES}}^{i p}$ is the battery voltage which is a function of the battery state of charge $\operatorname{SoC}_{\mathrm{DES}}^{i p}, C_{\mathrm{DES}}^{i p}$ is the battery capacity, and $\mathbf{p}_{\mathrm{DES}}^{i p}$ is a set of battery modeling parameters. Details of the battery modeling approach, including the efficiency and the Depth of Discharge (DoD) considerations, can be found in a previous work [21] of the authors. The average energy efficiency of a lead acid battery, obtained by multiplying the voltage efficiency and the coulomb efficiency may typically vary between $77 \%$ and $81 \%$ [22].

\section{B. Dynamic Modeling and Controller Design for Distributed Storage}

A dynamic control model is developed to investigate the proposed strategy in the presence of physical device time lags. In the dynamic control model, a PI controller is used for storage power control, and the storage unit, DC/DC converters and inverter are represented by first order time lags, as shown in Fig. 7.

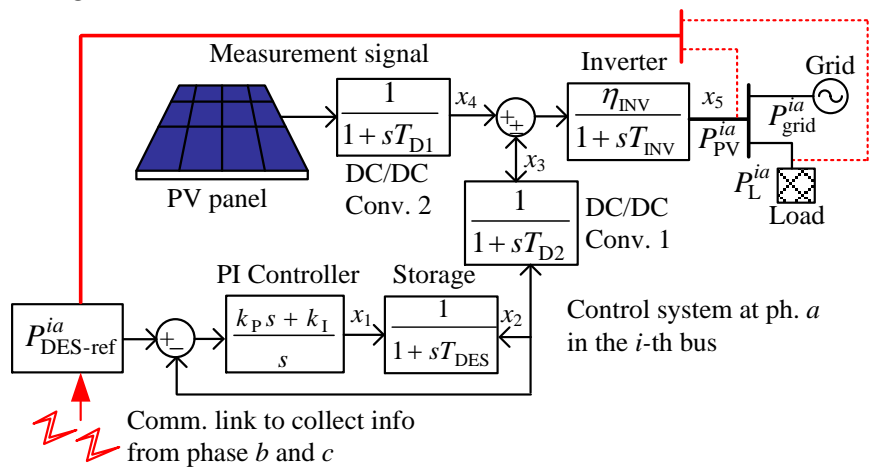

Fig. 7. Dynamic model of the storage control system for power balancing.

The state-space model of the dynamic system, shown in Fig. 7, constructed using the state variables corresponding to the storage power control loop ( $x_{1}$ : PI controller output, $x_{2}$ : $\left.P_{\mathrm{DES}}\right), \mathrm{DC} / \mathrm{DC}$ converter outputs $\left(x_{3}, x_{4}\right)$ and inverter output $\left(x_{5}\right)$ is given in (7). The reference for the PI controller, $P_{\mathrm{DES}-\text { ref }}$ is obtained using (3)-(5).

$$
\begin{gathered}
\mathbf{x}=\left[\begin{array}{lllll}
x_{1} & x_{2} & x_{3} & x_{4} & x_{5}
\end{array}\right]^{T}, \mathbf{u}=\left[\begin{array}{cccc}
P_{\mathrm{DES}-\mathrm{ref}} & P_{\mathrm{DC}}
\end{array}\right]^{T} \\
\mathbf{A}=\left[\begin{array}{ccccc}
-\frac{k_{\mathrm{P}}}{T_{\mathrm{DES}}}\left(\frac{k_{\mathrm{P}}}{T_{\mathrm{DES}}}-k_{\mathrm{I}}\right) & 0 & 0 & 0 \\
\frac{1}{T_{\mathrm{DES}}} & -\frac{1}{T_{\mathrm{DES}}} & 0 & 0 & 0 \\
0 & \frac{1}{T_{\mathrm{D} 1}} & -\frac{1}{T_{\mathrm{D} 1}} & 0 & 0 \\
0 & 0 & 0 & -\frac{1}{T_{\mathrm{D} 2}} & 0 \\
0 & 0 & \frac{\eta_{\mathrm{INV}}}{T_{\mathrm{INV}}} & \frac{\eta_{\mathrm{INV}}}{T_{\mathrm{INV}}} & -\frac{1}{T_{\mathrm{INV}}}
\end{array}\right] \\
\mathbf{B}=\left[\begin{array}{ccccc}
k_{\mathrm{I}} & 0 & 0 & 0 & 0 \\
0 & 0 & 0 & \frac{1}{T_{\mathrm{D} 1}} & 0
\end{array}\right]^{T}
\end{gathered}
$$

where, $T_{\mathrm{DES}}$ is the storage time constant, $T_{\mathrm{D} 1}$ and $T_{\mathrm{D} 2}$ are the time constants of the DC/DC converters, $T_{\mathrm{INV}}$ is the inverter 
time constant and $\eta_{\mathrm{INV}}$ is the inverter efficiency that can be obtained from manufacturer datasheet based on the operating condition.

The rise time $\left(\tau_{\mathrm{r}}\right)$ and overshoot (OS) of the closed loop system formed by the controller and the storage device are used as the design criteria for obtaining the controller parameters. The rise time needs to be low so that the storage device is able to provide a fast and appropriate balancing effect. Simultaneously, the overshoot needs to be within a satisfactory limit. The rise time is chosen to be slightly higher than $T_{\mathrm{DES}}$ and the overshoot is chosen to be kept below $15 \%$.

The closed loop transfer function $G_{\mathrm{c}}(s)$ of the controller and storage combined system in terms of the storage time constant $T_{\mathrm{DES}}$, and the proportional and integral gains $\left(k_{\mathrm{P}}, k_{\mathrm{I}}\right)$ is given below.

$$
G_{c}(s)=\frac{\left(\frac{k_{\mathrm{P}}}{T_{\mathrm{DES}}}\right) s+\frac{k_{\mathrm{I}}}{T_{\mathrm{DES}}}}{s^{2}+\left(\frac{1+k_{\mathrm{P}}}{T_{\mathrm{DES}}}\right) s+\frac{k_{\mathrm{I}}}{T_{\mathrm{DES}}}}
$$

According to the dynamics of second order systems, the natural frequency $\omega_{n}$ and the damping ratio $\zeta$ can be related to the storage and controller parameters as given in (9).

$$
\omega_{n}=\sqrt{\frac{k_{\mathrm{I}}}{T_{\text {Sto }}}}, \zeta=\frac{1+k_{\mathrm{P}}}{2 \omega_{n} T_{\text {Sto }}}
$$

The value of $\zeta$ is determined from the specified overshoot criterion using the following expression [23].

$$
\zeta=-\frac{\log (\mathrm{OS})}{\sqrt{\pi^{2}+[\log (\mathrm{OS})]^{2}}}
$$

From the obtained value of $\zeta, \omega_{n}$ can be determined using the normalized rise time versus damping ratio characteristic presented in [23]. The values of $\omega_{n}$ and $\zeta$ are then used to obtain $k_{\mathrm{P}}$ and $k_{\mathrm{I}}$, using (9).

Modern battery energy storage devices, such as Valve Regulated Lead Acid (VLRA) batteries, Li-Ion batteries, have response time in the order of milliseconds. The VLRA battery used in this paper is assumed to have a time constant $\left(T_{\mathrm{DES}}\right)$ of 6 millisecond according to [24,25]. Based on the value of $T_{\mathrm{DES}}$, the rise time $\left(\tau_{\mathrm{r}}\right)$ criterion is set at 8 milliseconds and the overshoot (OS) criterion is set at $10 \%$. Then using the approach stated above, the controller parameters are obtained, as listed in Table I. The time constants of the DC/DC converters and inverter are derived from typical values given in $[26,27]$ and presented in Table I. The root-locus plot in Fig. 8(a) shows that using the controller gains listed in Table I, the closed loop poles are located on the line corresponding to $\zeta=$ 0.56 , which corresponds to $11 \%$ overshoot. The open loop frequency response characteristic in Fig. 8(b) shows a phase margin of $60.8^{\circ}$ indicating a stable loop.

TABLE I

DyNAMIC MOdel AND CONTROLLeR PARAMETERS

\begin{tabular}{l||c}
\hline \multicolumn{1}{c|}{ Parameter } & \multicolumn{1}{c}{ Value } \\
\hline \hline$T_{\mathrm{DES}}, T_{\mathrm{D} 1}, T_{\mathrm{D} 2}, T_{\mathrm{INV}}$ & $6 \mathrm{~ms}, 6 \mathrm{~ms}, 6 \mathrm{~ms}, 12 \mathrm{~ms}$ \\
\hline$K_{\mathrm{P}}, K_{\mathrm{I}}$ & $0.4808,261.4594$ \\
\hline \hline
\end{tabular}
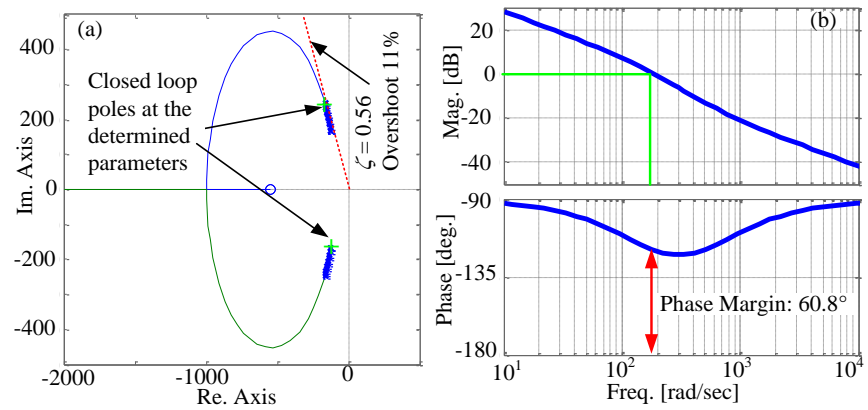

Fig. 8. Controller performance. (a) Root locus. (b) Frequency response.

\section{A Control Strategy for Charging/Discharge Operation}

The control strategy for charging/discharging of the distributed storage connected at a given $i$-th bus and $p$-th phase is shown in Fig. 9 using a flowchart for a given $k$-th time instant.

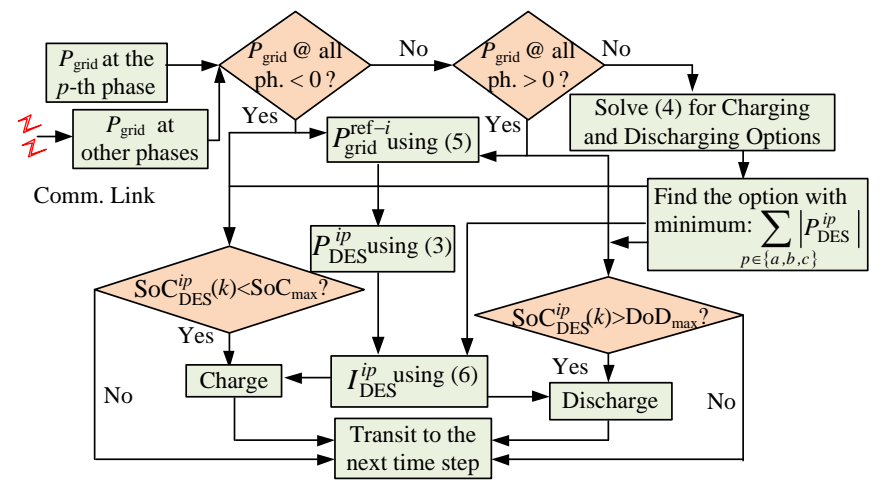

Fig. 9. The balancing control strategy with distributed storage.

The amount of the grid power exchange $\left(P_{\text {grid }}\right)$ at the $(p$-th) phase (from measurement) and the other two phases is monitored (through communication) at each instant of time. The measurements signals necessary for this strategy is the load and PV generation at each of the PV connection points that would be available from the standard monitoring, control and protection systems of grid-tied inverter units. If $P_{\text {grid }}$ at all the phases are less than zero, this indicates a reverse power flow (during high PV period) at the phases and therefore power balancing is performed by charging operation. The appropriate charging rates for the balancing operation are obtained using (6), based on the values obtained from (3) and (5). Then the charging operation is performed if the present SoC is less than the maximum allowable state of charge of the battery $\left(\mathrm{SoC}_{\max }\right)$. If $P_{\text {grid }}$ at all the phases are higher than zero, this indicates a forward power flow (during low or no PV period) at the phases and therefore power balancing is performed by discharging operation. The discharging rates are obtained using (3), (5) and (6), and the discharge operation is performed if the present $\mathrm{SoC}$ is higher than $\mathrm{DoD}_{\max }$ to ensure that it is not allowed to be fully discharged (empty).

If $P_{\text {grid }}$ at the phases have both forward and reverse power flow, then (4) is solved to find the total power for charging and discharging options. Then the balancing operation is performed by the option that requires the minimum amount of total power.

The proposed strategy is developed based on a distributed control approach where the distributed storage devices will 
operate depending on local load and PV output information at the same bus. The communication requirement would be minimal and communication with any central control system will not be necessary.

\section{APPLICATION EXAMPLES}

To analyze the performance of the proposed strategy in a distribution network environment, a low voltage (LV) distribution feeder extracted from the New South Wales distribution system is considered. All the households in the $\mathrm{LV}$ feeder are assumed to have rooftop solar PV units with integrated battery storage devices. For the analysis of distributed storage based strategy, 3 to $8 \mathrm{kWh}$ VLRA battery (typical Australian size for residential or small PV systems) is used depending on the load and PV size at different phases. In spite of the weight and dimensional constraints (147 kg, $665 \times 217 \times 412 \mathrm{~mm}^{3}$ for a typical $3.48 \mathrm{kWh}$ VRLA battery [28]), this type of energy storage devices are being integrated with the home PV systems. According to manufacturer test reports [29], the charging/discharging rates of VRLA batteries can be varied in a wide range from $0.05 \mathrm{C}$ to $4 \mathrm{C}$, where $\mathrm{C}$ is the capacity and therefore would be able to satisfy the requirements of a wide range of charging/discharging power.

Before testing the proposed approach under a real load demand and PV generation profile over a daylong period, an investigation is performed to observe how this mitigation technique provides balancing effect and reduces neutral current and NGV at a given time instant. The dynamic control model of the integrated PV and energy storage system is used for this purpose. Power balancing by the charging operation is shown in Fig. 10 using a time instant during the midday.
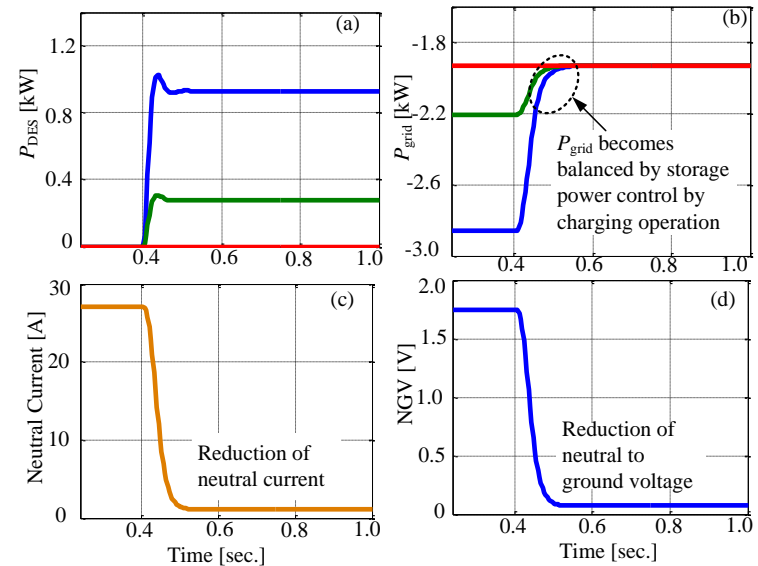

Fig. 10. Power balancing by charging operation (a) $P_{\mathrm{DES}}$. (b) $P_{\text {grid }}$ (c) Neutral current. (d) NGV.

Initially, the storage control is deactivated up to $0.4 \mathrm{sec}$ to show the impact of unbalanced load and PV generation on neutral current and neutral to ground voltage (NGV). This causes the storage power consumption to remain zero before $0.4 \mathrm{sec}$, as shown in Fig. 10(a), and due to the presence of load and PV unbalances, unbalance is created in $P_{\text {grid }}$, as presented in Fig. 10(b). Therefore, a high neutral current and a high NGV are produced, as shown in Fig. 10(c) and 10(d), respectively. At $0.4 \mathrm{sec}$, the storage power control is activated and an appropriate power balancing action takes place in several milliseconds, which is observed in storage power consumption profiles presented in Fig. 10(a). This results in balancing the amount of $P_{\text {grid, }}$ and therefore, a reduction in neutral current and voltage, as shown in Fig. 10(b), (c), and (d), respectively.

Power balancing by the discharge operation of the energy storage devices during evening peak load period is shown in Fig. 11. Similar to the charging operation in Fig. 10, the storage control is deactivated up to $0.4 \mathrm{sec}$, which results in an unbalance in $P_{\text {grid }}$ created by supplying unbalanced loads, as shown in Fig. 11(a) and (b), respectively. The neutral current in Fig. 11(c) produced by the unbalanced load creates a significant level of NGV as shown in Fig. 11(d). At $0.4 \mathrm{sec}$, the storage power control is activated and an appropriate balancing effect is provided by discharging the stored energy which balances the $P_{\text {grid, }}$ and reduces neutral current and NGV, as shown in Fig. 11(b), (c), and (d), respectively. Results presented in Fig. 10 and 11 verify the ability of the proposed approach to mitigate neutral current and NGV problems under physical device time lags. Energy storage devices with low response time and an appropriate design of the controller would be necessary to effectively utilize the proposed approach.
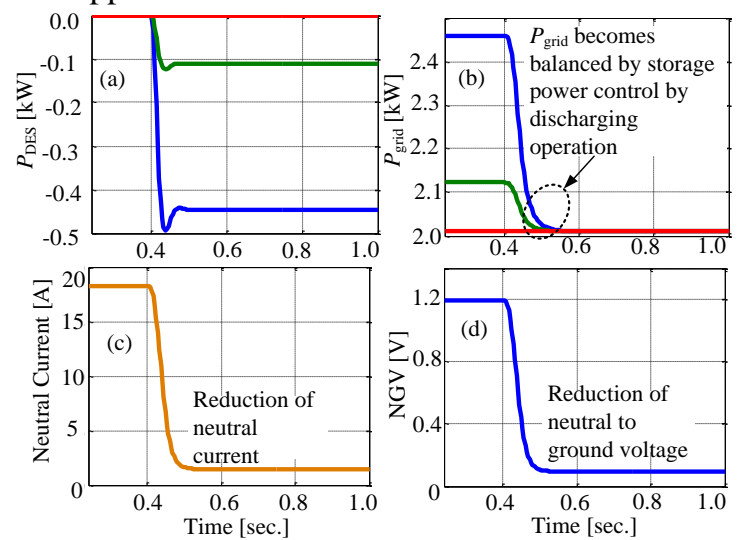

Fig. 11. Power balancing by discharging operation (a) $P_{\mathrm{DEs}}$. (b) $P_{\text {grid. }}$ (c) Neutral current. (d) NGV.

The performance of the proposed strategy to mitigate neutral current and NGV problems under daylong variations of PV output and load is verified using quasi steady state simulations using 1-minute resolution data. The solar PV data is obtained from the Renewable Energy Integration Facility at Commonwealth Scientific and Industrial Research Organisation (CSIRO) Energy Technology in Australia and the load data is derived from an Australian distribution utility.

The distributed storage devices installed in the test LV feeder is controlled according to the proposed power balancing algorithm. $P_{\text {grid }}$ profiles for the three phases in the substation-end bus of the test feeder and the reference power exchange with the grid determined based on the $P_{\text {grid }}$ profiles using (3) to (5) are shown in Fig. 12 (a). During the periods of mixed $P_{\text {grid }}$ values (+ve and -ve), as marked using blue circles, the storage devices are controlled using (4) as shown in the flowchart of Fig. 9. The SoC profile of the storage device in phase $a$ of the substation-end bus is shown in Fig. 12(b); the $\mathrm{DoD}_{\max }(35 \%)$ and $\mathrm{SoC}_{\max }(100 \%)$ limits are checked while performing the charging/discharging operation.

The power delivered or absorbed by the distributed storage 
devices at the substation-end bus of the LV feeder is shown in Fig. 13. The shaded areas indicate the energy used in the charging/discharging operation. The time corresponding to the charging/discharging operations are also identified over the 24 hour period in Fig. 13. The energy stored during the charging operation over the daytime is discharged during the nighttime. Depending on the amount of stored energy, the period of the discharge operation can be determined for a given load demand profile. If the stored energy is adequate, the balancing support can be continued over the late night to early morning, as shown in Fig. 13. However, if the amount of stored energy is not adequate, the balancing support could only be provided during the peak load period, when the neutral current and the NGV problems are most severe due to a high amount of load unbalance.
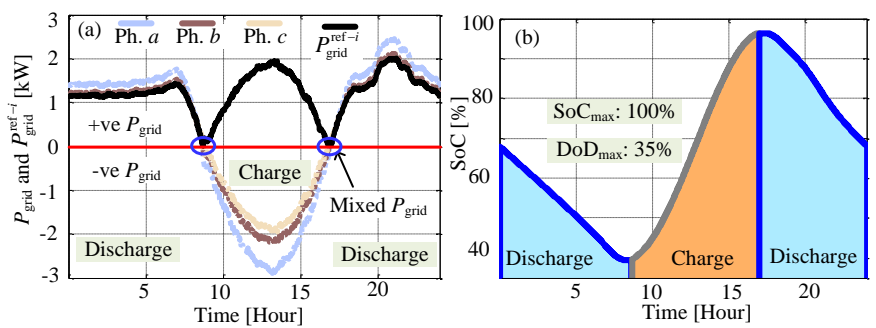

Fig. 12. Charge and discharge operation of energy storage. (a) $P_{\text {grid }}$ and $P_{\text {grid }}^{\text {ref } i}$ profile. (b) SoC profile during charge and discharge operation.

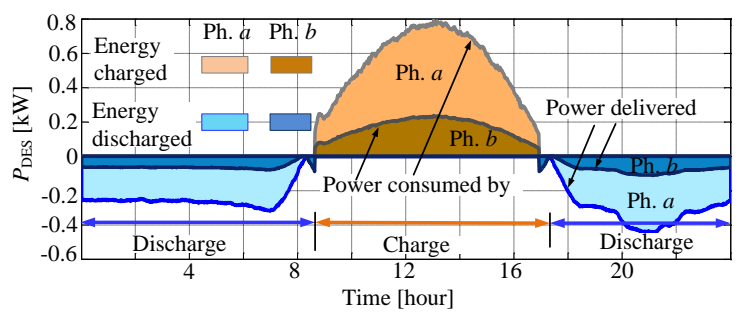

Fig13. Power absorbed and delivered by distributed storage devices.

Fig. 14(a) shows the feeder current flow without any mitigation. It is observed that during midday and also during the evening peak period, the currents in the three phases are unbalanced, with a Current Unbalance Factor (CUF) of $14 \%$ and $10 \%$, respectively. These unbalanced phase currents produce a high neutral current.

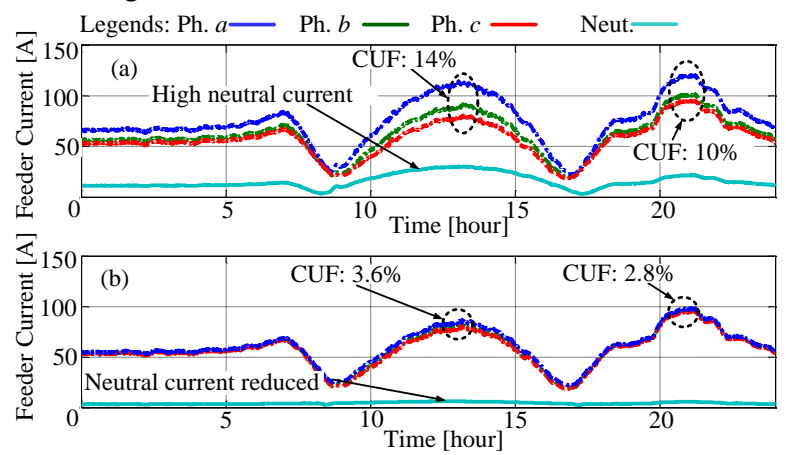

Fig. 14. Phase and neutral current flow through the test feeder; (a) without mitigation; (b) with mitigation by distributed storage devices.

The current unbalance is reduced by the balancing action of the storage units (CUF value reduced to $3.6 \%$ and $2.8 \%$, respectively) and, the neutral current is also reduced as shown in Fig. 14(b). The reduction of neutral current will help to mitigate the neutral potential rise problem. The NGV profiles at the substation-end bus and at the remote-end bus of the LV feeder without balancing action are compared in Fig. 15 with the NGV profiles at the same buses with balancing action. It is observed that with the power balancing operation, the NGV values are reduced significantly during midday PV peak and also during the evening peak load period. For reference, the NGV profiles at the other buses of the LV feeder have also been included in Fig. 15. It is found that the proposed mitigation strategy has reduced the $\mathrm{NGV}$ values all over the $\mathrm{LV}$ feeder below the limit of $0.5 \mathrm{~V}$.

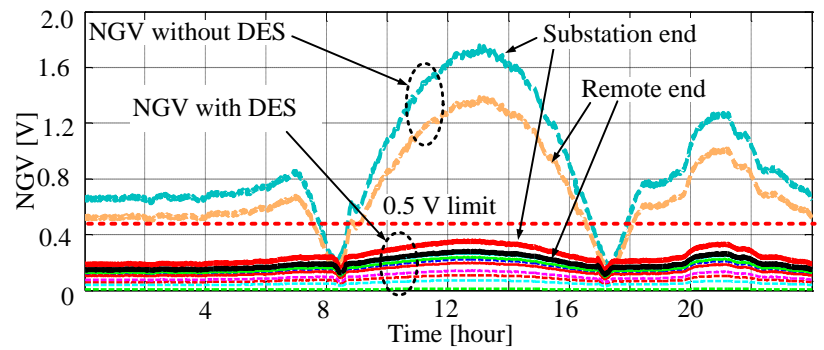

Fig. 15. NGV with the mitigation by distributed energy storage.

Previous results show how the proposed strategy mitigates neutral current and neutral potential rise problem over a daylong period where the PV output has no significant fluctuation. If a PV output fluctuates due to cloud passing, the storage device will need to shift the operation from charging to discharging. The applicability of the proposed approach under a fluctuating PV output scenario is investigated below. First, a quasi steady state analysis is performed using a 700 second long PV output profile captured by National Renewable Energy Laboratory (NREL) [30] from Hawaii Island. Then a dynamic simulation is performed using the dynamic model developed in this paper, as shown in Fig. 7, to investigate how the proposed approach performs in providing the appropriate mitigation action under physical device time delays. As the resolution of the PV data is 1-second, the reference value of the controller was updated every second.

The measurements signals necessary for this strategy is the load and PV generation at each of the PV connection points that would be available from the standard monitoring, control and protection systems of grid-tied inverter units. Therefore, no additional cost will be associated with the measurement.

The results of quasi steady analysis are presented in Fig. 16. The unbalances in $P_{\text {grid }}$ due to load and PV unbalance is shown in Fig. 16(a), where fluctuations in $P_{\text {grid }}$ are observed from $200 \mathrm{sec}$ to $325 \mathrm{~second}$, caused by PV output fluctuation. Using the proposed strategy, storage devices are controlled to consume and deliver the appropriate amount of balancing power, as shown in Fig. 16(b), by charging and discharging operation. Therefore, the $P_{\text {grid }}$ profiles become balanced, as observed in Fig. 16(c). Providing the necessary balancing effect by the storage devices results in reducing the neutral current and neutral potential, as observed in Fig. 17(a) and (b), respectively.

For the dynamic simulation of the proposed strategy under a fluctuating PV output environment, a 6-second long segment from the 700-second profile used in Fig. 16 is considered. The segment is identified in Fig. 16(a) using a dashed rectangle, which includes both injection into the grid and consumption 
from the grid, therefore requiring a charging and a discharging operation.
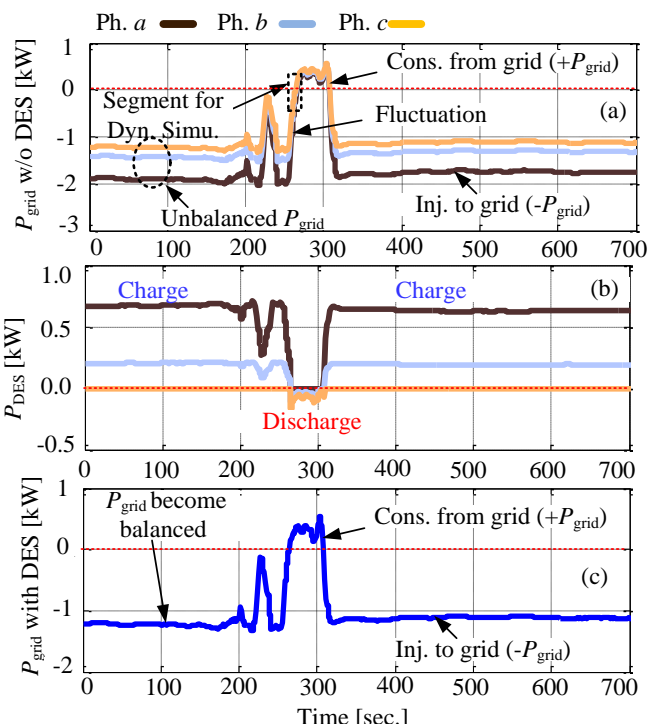

Fig. 16. Performance of the proposed approach under fluctuating PV output. (a) $P_{\text {grid }}$ without DES (b) $P_{\mathrm{DES}}$ (c) $P_{\text {grid }}$ with DES.
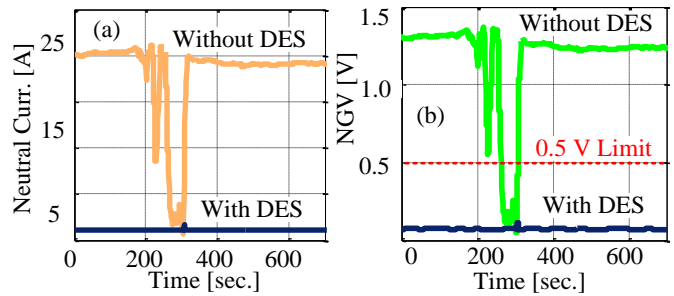

Fig. 17. Neutral current and NGV with and without DES during a PV output fluctuation event. (a) Neutral current reduction. (b) NGV reduction

Without the control of the storage devices, $P_{\text {grid }}$ is unbalanced, as shown in Fig. 18(a). With proposed control algorithm, $P_{\mathrm{DES}}$ at all the phases is controlled to provide an appropriate balancing action. From 0 to 2 seconds, balancing is performed by charging operation, and after 2 second, the $P_{\text {grid }}$ becomes positive due to reduction in PV output caused by cloud passing. Therefore, $P_{\mathrm{DES}}$ control is shifted from charging to discharging for providing the appropriate balancing effect. As a result, $P_{\text {grid }}$ at all the phases become balanced, as observed in Fig. 18(c).

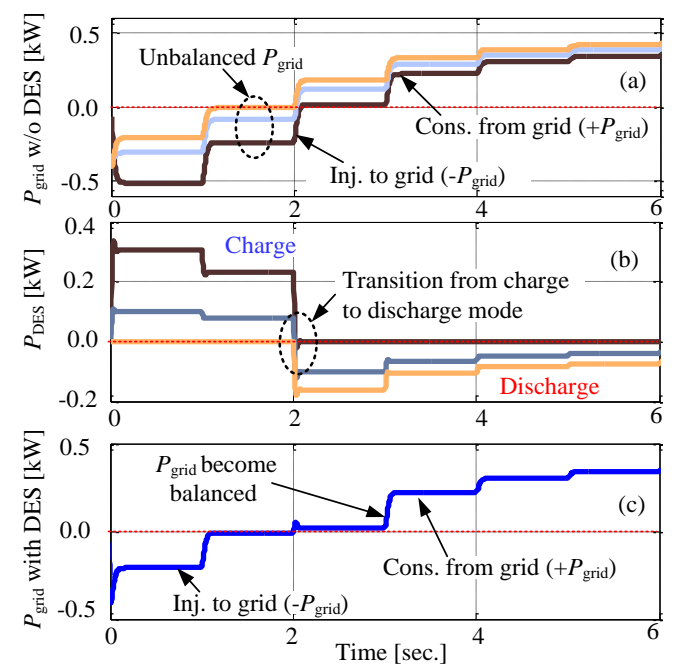

Fig. 18. Dynamic performance of the proposed approach under fluctuating PV output. (a) $P_{\text {grid }}$ without DES (b) $P_{\text {DES. }}$ (c) $P_{\text {grid }}$ with DES.
The neutral current and NGV profiles obtained from the dynamic simulation are presented in Fig. 19 that shows significant reductions in the neutral current and neutral potential as a result of controlling the distributed storage devices using the proposed strategy.
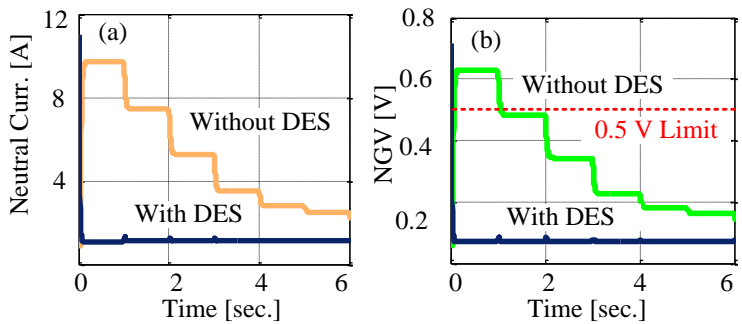

Fig. 19. Dynamic response of neutral current and NGV obtained using the dynamic model. (a) Neutral current. (b) NGV.

The proposed strategies perform the mitigation of the neutral current and NGV problems by storing surplus energy during PV peak period and releasing the stored energy during the evening peak load. In addition to mitigating the NGV problem, this operation brings the benefit of partially mitigating voltage rise impact of PV and also supports the evening peak load. Market analysts are expecting a tenfold increase in the global market of storage devices for small residential PV systems [31]. Utilities in Australia are promoting the utilization of energy storage devices. In some cases, its use is becoming mandatory because Australian utilities are putting in standards that will only allow an automatic approval of PV systems below $30 \mathrm{~kW}$ if it is equipped with any device that prevents it from exporting power to the grid [32] - such a device would essentially be an energy storage. With the availability of PV integrated energy storage devices and communication systems at each of the customer premises, this paper only proposes a strategy to increase the benefit of the already available assets by utilizing the energy storage device for alleviating the neutral voltage rise.

\section{CONCLUSIONS}

This paper has developed a novel strategy for the mitigation of the neutral current and neutral potential problems in 4-wire multi-grounded LV networks under unbalanced allocation of rooftop solar PV using distributed energy storage. The controllability of energy storage devices has been exploited to provide a dynamic balancing effect under the combined unbalance of variable load and solar PV generation to mitigate the neutral current and NGV problems. An algorithm has been developed based on constrained minimization technique to balance the net power exchange with the grid utilizing the minimum amount of storage power. A control strategy has been developed based on the proposed balancing algorithm to control the energy storage devices to provide an appropriate balancing effect under varying load and PV unbalance. Analysis performed using an Australian distribution feeder demonstrates, that the proposed strategy is able to reduce the large neutral current created by the combined load and PV unbalance and hence reduce the NGV 
level to within the acceptable limit. As the energy storage devices and the power electronic converters in the PV systems have time delays, a dynamic control model of the PV storage integrated system is developed and a controller is designed to investigate the performance of the proposed strategy under physical time lags. It is observed that with a proper selection of energy storage and an appropriately tuned controller, the proposed strategy can mitigate the neutral current and NGV problems in the order of milliseconds.

\section{REFERENCES}

[1] J. C. Balda, A. R. Oliva, D. W. McNabb, and R. D. Richardson, "Measurements of neutral currents and voltages on a distribution feeder," IEEE Transactions on Power Delivery, vol. 12, pp. 1799-1804, 1997.

[2] J. Werda, J. Chan, and P. Freeman. Electrical Earthing - Risk Management Strategies for the Water Industry, presented at the 2nd Annual Water Industry Engineers and Operators Conference, Newcastle, NSW, Australia, 8-10 April, 2008. [Online]. Available: http://www.wioa.org.au/conference_papers/08_nsw/documents/johnwe rda.pdf

[3] T. M. Gruzs, "A survey of neutral currents in three-phase computer power systems," IEEE Transactions on Industry Applications vol. 26, pp. 719-725, 1990.

[4] Power Quality - The Basics [Online]. Available: http://www.psihq.com/iread/pqbasics.htm

[5] D. W. Zipse, "The hazardous multigrounded neutral distribution system and dangerous stray currents," in IEEE Industry Applications Society 50th Annual Petroleum and Chemical Industry Conference, Houston, TX, USA, 15-17 Sep., 2003, pp. 23-45.

[6] Effects of Electrcal Voltage/Current on Farm Animals [Online]. Available: http://naldc.nal.usda.gov/download/CAT92970513/PDF

[7] Elevated Neutral-to-Earth Voltage, Urban Contact Voltage and Other Perceptibility Concerns for Humans and Animals [Online]. Available: http://strayvoltage.epri.com/index.asp

[8] G. Ahmadi and S. M. Shahrtash, "Neutral to earth voltage reduction methods in three-phase four wire distribution systems," in International Conference on Electrical and Electronics Engineering, Bursa, Turkey, 5-8 Nov., 2009, pp. I-134-I-138.

[9] T. C. Surbrook, N. D. Reese, and A. M. Kehrle, "Stray Voltage: Sources and Solutions," IEEE Transactions on Industry Applications, vol. IA-22, pp. 210-215, 1986.

[10] B. Jamali, R. Piercy, and P. Dick. Stray Voltage Mitigation [Online]. Available: http://www.ontarioenergyboard.ca/OEB/_Documents/EB2007-0709/report_Kinectrics_20080530.pdf

[11] S. Xiangjing, M. A. Masoum, and P. J. Wolfs, "Optimal PV Inverter Reactive Power Control and Real Power Curtailment to Improve Performance of Unbalanced Four-Wire LV Distribution Networks," IEEE Transactions on Sustainable Energy vol. 5, pp. 967-977, 2014.

[12] P. Acuna, L. Moran, M. Rivera, J. Dixon, and J. Rodriguez, "Improved Active Power Filter Performance for Renewable Power Generation Systems," IEEE Transactions on Power Electronics vol. 29, pp. 687694, 2014.

[13] G. Mokhtari, A. Ghosh, G. Nourbakhsh, and G. Ledwich, "Smart Robust Resources Control in LV Network to Deal With Voltage Rise Issue," IEEE Transactions on Sustainable Energy vol. 4, pp. 10431050, 2013.

[14] K. H. Chua, L. Yun Seng, P. Taylor, S. Morris, and W. Jianhui, "Energy Storage System for Mitigating Voltage Unbalance on LowVoltage Networks With Photovoltaic Systems," IEEE Transactions on Power Delivery, vol. 27, pp. 1783-1790, 2012.

[15] M. J. E. Alam, K. Muttaqi, and D. Sutanto, "Effectiveness of Traditional Mitigation Strategies for Neutral Current and Voltage Problems under High Penetration of Rooftop PV," in IEEE PES General Meeting, Vancouver, British Columbia, Canada, Jul. 21 - 25, 2013.

[16] A. D. Kolagar, P. Hamedani, and A. Shoulaie, "The effects of transformer connection type on voltage and current unbalance propagation," in Power Electronics and Drive Systems Technology, Tehran, Iran, 15-16 Feb., 2012, pp. 308-314.

[17] M. J. E. Alam, K. M. Muttaqi, and D. Sutanto, "A Three-Phase Power Flow Approach for Integrated 3-Wire MV and 4-Wire Multigrounded
LV Networks With Rooftop Solar PV," IEEE Transactions on Power Systems, vol. 28, pp. 1728-1737, 2013.

[18] P. Kansal and A. Bose, "Bandwidth and Latency Requirements for Smart Transmission Grid Applications," IEEE Transactions on Smart Grid, vol. 3, pp. 1344-1352, 2012.

[19] Y. Ye, Q. Yi, H. Sharif, and D. Tipper, "A Survey on Smart Grid Communication Infrastructures: Motivations, Requirements and Challenges," IEEE Communications Surveys \& Tutorials, vol. 15, pp. 5-20, 2013

[20] A. Aggarwal, S. Kunta, and P. K. Verma, "A proposed communications infrastructure for the smart grid," in Innovative Smart Grid Technologies (ISGT), Gaithersburg, MD, USA, 19-21 Jan. 2010, pp. 15.

[21] M. J. E. Alam, K. M. Muttaqi, and D. Sutanto, "Mitigation of Rooftop Solar PV Impacts and Evening Peak Support by Managing Available Capacity of Distributed Energy Storage Systems," IEEE Transactions on Power Systems, vol. 28, pp. 3874-3884, November 2013.

[22] G. M. Masters, Renewable and Efficient Electric Power Systems. New Jersey: Wiley-IEEE Press, 2004.

[23] N. S. Nise, Control Systems Engineering, 4th Edition ed.: Wiley, 2004.

[24] H. Chen, T. N. Cong, W. Yang, C. Tan, Y. Li, and Y. Ding, "Progress in electrical energy storage system: A critical review," Progress in Natural Science, vol. 19, pp. 291-312, 2009.

[25] B. Espinar and D. Mayer. The role of energy storage for mini-grid stabilization [Online]. Available: http://www.iea-pvpstask11.org/HTMLobj-187/Act_24_Final.pdf

[26] K. Clark, R. A. Walling, and N. W. Miller, "Solar photovoltaic (PV) plant models in PSLF," in IEEE Power and Energy Society General Meeting, Detroit, MI, USA, 24-29 July 2011, pp. 1-5.

[27] Y. Meng and W. Xiaoyu, "Dynamic modeling of grid integrated solar and battery energy system for cloud transient impact study," in IEEE Energytech, Cleveland, OH, USA, 21-23 May 2013, pp. 1-6.

[28] Absolyte GP Photovoltaic and Alternative Energy Section 62.612013 09 [Online]. Available: http://www.exide.com/Media/files/Downloads/IndustAmer/Section\%20 62_61\%202013-

09\%20Absolyte\%20GP\%20Photovoltaic\%20\%26\%20Alternative $\% 20$ Energy\%20English.pdf

[29] C\&D Technologies Technical Bulletin 41-7135: Capacity Testing of VRLA Batteries (20 to 200 Ampere-Hours Capacity) [Online]. Available: http://www.cdtechno.com/pdf/ref/41_7135_0412.pdf

[30] NREL. 1-Second Global Horizontal Irradiance Oahu, Hawaii [Online]. Available: http://www.nrel.gov/midc/oahu_archive/

[31] T. Hanes and S. Ullrich. (21 November 2014). Batteries for private PV systems tipped to jump 10-fold [Online]. Available: http://reneweconomy.com.au/2014/batteries-private-pv-systems-tippedjump-10-fold-21260

[32] G. Parkinson. (27 June 2014). Utilities move to kick rooftop solar off the grid [Online]. Available: http://reneweconomy.com.au/2014/utilities-move-to-kick-rooftop-solaroff-the-grid- 15250

\section{BIOGRAPHIES}

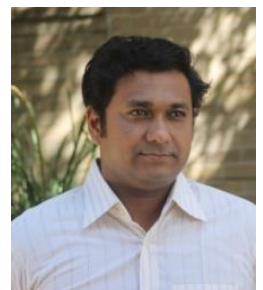

M J E Alam (M'14) received B.Sc. and M.Sc Degree in Electrical and Electronic Engineering from Bangladesh University of Engineering and Technology, Dhaka, Bangladesh, in 2005 and 2009, respectively. In 2014, he obtained $\mathrm{PhD}$ degree from the University of Wollongong, New South Wales, Australia. His research interest includes modeling and analysis of power systems considering the impacts of distributed and renewable energy resources. He has also worked in the electric power industry in Bangladesh.

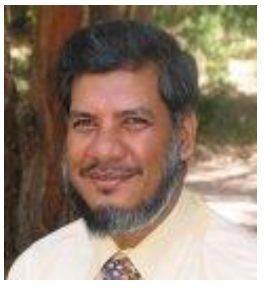

K M Muttaqi (M'01, SM'05) received the B.Sc degree in electrical and electronic engineering from Bangladesh University of Engineering and Technology (BUET), Bangladesh in 1993, the M.Eng.Sc. degree in electrical engineering from University of Malaya, Malaysia in 1996 and the $\mathrm{Ph} . \mathrm{D}$. degree in Electrical Engineering from Multimedia University, Malaysia in 2001. Currently, he is an Associate Professor at the School of 
Electrical, Computer, and Telecommunications Engineering, and member of Australian Power Quality and Reliability (APQRC) at the University of Wollongong, Australia. He was associated with the University of Tasmania, Australia as a Research Fellow/Lecturer/Senior Lecturer from 2002 to 2007, and with the Queensland University of Technology, Australia as a Research Fellow from 2000 to 2002. Previously, he also worked for Multimedia University as a Lecturer for three years. He has more than 18 years of academic experience and has authored or coauthored around 200 papers in international journals and conference proceedings. His research interests include distributed generation, renewable energy, electrical vehicles, smartgrid, power system planning and control. Dr. Muttaqi is an Associate Editor of the IEEE TRANSACTIONS ON INDUSTRY APPLICATIONS.

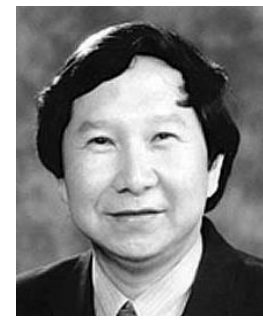

D Sutanto (SM'89) obtained his BEng. (Hons) and $\mathrm{PhD}$ from the University of Western Australia. He is presently the Professor of Power Engineering at the University of Wollongong, Australia. His research interests include power system planning, analysis and harmonics, FACTS and Battery Energy Storage systems. He is a Senior Member of IEEE. He is currently the IEEE IAS Area Chair for Region 10 (South East Asia and Pacific). 